\title{
Severe varicella in persons vaccinated with varicella vaccine (breakthrough varicella): a systematic literature review
}

\author{
Jessica Leung, MPH $^{1,{ }^{*}}$, Karen R. Broder, MD ${ }^{2}$, and Mona Marin, MD ${ }^{1}$ \\ ${ }^{1}$ Epidemiology Branch, Division of Viral Diseases, National Center for Immunization and \\ Respiratory Diseases, Centers for Disease Control and Prevention, Atlanta, GA, USA \\ 2Immunization Safety Office, Division of Healthcare Quality Promotion, National Center for \\ Emerging and Zoonotic Infectious Diseases, Centers for Disease Control and Prevention, Atlanta, \\ GA, USA
}

\section{Abstract}

INTRODUCTION-Varicella vaccines are highly effective at preventing disease, but varicella may occur among vaccinated persons (termed breakthrough varicella). Breakthrough varicella is generally mild, but severe cases have been reported. The objective of this review is to describe severe breakthrough varicella.

\begin{abstract}
AREAS COVERED-We conducted a systematic review of articles published during 19742016. A total of 34 articles were included in our review: 21 described breakthrough varicella with disseminated varicella-zoster virus (VZV) infection with other organ involvement in addition to skin (none among two-dose vaccinees); 9 described hospitalized breakthrough varicella without mention of other organ involvement in addition to skin (of which 2 reported 4 two-dose vaccinees); and 4 described both. A total of 52-60 unique breakthrough varicella cases with disseminated VZV infection with other organ involvement in addition to skin reported with the following complications, not mutually exclusive: pneumonia ( $\mathrm{n}=8-9$ cases), neurologic ( $\mathrm{n}=18-24$ cases), hematologic ( $n=10-11$ cases), ocular ( $n=5$ cases), renal ( $n=2$ cases), hepatic ( $n=3$ cases), secondary infection with bacteremia or sepsis ( $\mathrm{n}=8$ cases), and other complication ( $\mathrm{n}=4$ cases). There were 6 cases of fatal breakthrough varicella.
\end{abstract}

EXPERT COMMENTARY - With >31 million doses distributed annually worldwide since 2007, severe breakthrough varicella can occur but they appear to be uncommon.

\section{Keywords}

Varicella; vaccine; varicella-zoster virus (VZV); chickenpox; breakthrough; modified varicella; literature review; varicella vaccine failure

"Corresponding author: Jessica Leung, MPH, Centers for Disease Control and Prevention, 1600 Clifton Rd NE, MS A-34, Atlanta, GA 30333, Tel: 404-639-6067, Fax: 404-315-2486, JLeung@cdc.gov.

Disclosure Statement: The authors have no potential conflicts of interest to report.

Disclaimer: The findings and conclusions in this report are those of the authors and do not necessarily represent the official position of the Centers for Disease Control and Prevention, US Department of Health and Human Services. 


\section{Introduction}

Varicella (chickenpox) is a highly contagious disease caused by infection with varicellazoster virus (VZV). It presents as a generalized maculopapulovesicular rash with 250-500 lesions. Varicella is usually a self-limited disease ${ }^{1}$ but can cause significant morbidity and can lead to death, more commonly in immunocompromised patients, with skin and soft tissue infections, pneumonia, and encephalitis being the most frequent complications ${ }^{2,3}$. A varicella vaccine containing live-attenuated VZV (named Oka strain) was developed in Japan in the early $1970 \mathrm{~s}^{4}$. Several licensed varicella vaccines are available, all using liveattenuated VZV and all based on the Oka strain except the vaccine developed in South Korea $^{5}$. The vaccine is available as a single-antigen vaccine or in combination with measles, mumps, and rubella (MMRV). While the varicella vaccines are licensed worldwide, varicella vaccination is routinely recommended in a limited number of countries, either as a one or two-dose program ${ }^{6-8}$. Varicella vaccine is not recommended for most immunocompromised persons ${ }^{3}$. The United States was the first country to recommend a routine one-dose program in 1996 , then updated its recommendations to a routine two-dose program in $2006^{3}$.

Varicella vaccine is safe and effective at preventing varicella ${ }^{9-12}$. One dose is approximately $81 \%$ effective against any clinical disease and $>95 \%$ effective against combined moderate and severe varicella; the second dose provides additional protection against any varicella $^{9}, 13,14$. Varicella due to wild-type (WT)-VZV may occur in some persons vaccinated with varicella vaccine after exposure to WT-VZV and is termed breakthrough varicella (i.e., vaccine failure). Breakthrough varicella is generally milder in severity and duration than varicella in unvaccinated persons ${ }^{1}$; clinical symptoms usually consist of a rash with $<50$ lesions, predominately maculopapular although sometimes also with vesicular lesions ${ }^{15}$. However, there have been cases of breakthrough varicella that resulted in extensive rash, complications, and even death ${ }^{1,16}$. A systematic review of severe breakthrough varicella has not been performed to date. Understanding the range of severe complications of varicella among vaccinated persons is an important component of evaluating the varicella vaccination program. The objective of this literature review is to summarize and describe severe breakthrough varicella.

\section{Methods}

\subsection{Literature search}

We searched the PubMed Database for articles published from November 30, 1974, when information on varicella vaccine was first published, through August 1, 2016 using the following search terms: "(varicella or VZV or varicella-zoster virus or chickenpox) and ("breakthrough" or "vaccine failure" or "adverse event" or "vaccine effectiveness" or "vaccine safety" or "vaccinated" or "immunized")".

We reviewed each title and abstract to determine if the article might include data on breakthrough varicella cases. For articles that passed this initial screen, we reviewed the full text if the article was available in English; if the full text was not available in English, we used the information from the abstract. We also reviewed any additional references that 
described breakthrough varicella that were identified from the reference section of the articles reviewed and were not retrieved by the PubMed search.

\subsection{Definitions}

Breakthrough varicella is defined as varicella in a person who received at least one dose of varicella vaccine (single-antigen or MMRV vaccines) $\geq 42$ days before occurrence of symptoms, without other apparent cause ${ }^{17}$. Forty-two days, which equals two incubation periods for varicella ${ }^{1}$, is used to differentiate breakthrough cases from potential vaccinestrain varicella-like rashes. Post-licensure experience showed that vaccine-strain rashes occurred typically 21 days post-vaccination with a range of 5-42 days post-vaccination ${ }^{18}$.

For the purpose of our review, we defined severe breakthrough varicella as breakthrough varicella that resulted in 1) disseminated VZV infection with other organ involvement in addition to skin: pneumonia, complications related to nervous, hematologic, ocular, renal, and hepatic systems, sepsis and secondary infection resulting in bacteremia, and other complications, 2) any hospitalizations in addition to cases that met the first criterion, or 3) death. For articles that described severe breakthrough varicella, we abstracted, when available, data on number of breakthrough varicella cases reported, vaccine type, number of vaccine doses, age of vaccination, immune status or medication of case-patients, interval between vaccination and breakthrough varicella onset, complications, laboratory test results, and if hospitalized.

We excluded cases of 1) varicella due to vaccine-strain VZV, 2) re-activation (due to either vaccine-strain or WT-VZV) that were diagnosed as herpes zoster (shingles) or had a rash suggestive of herpes zoster, and 3) varicella-like illness in persons who had received herpes zoster vaccine, which is recommended for persons aged $\geq 60$ years in the United States ${ }^{19}$.

Two co-authors reviewed each article and classified severe breakthrough varicella according to our case definition (including classification by organ system category). When questions arose about the case classification, the articles were reviewed by and discussed with the third co-author (physician). A breakthrough case may have had multiple complications and be described in more than one organ system category. We present the number of articles that described severe breakthrough varicella and the number of unique cases. Due to the use of the same surveillance systems in some publications it is likely that some of the cases were presented more than once; thus, it was difficult to determine the total number of unique cases. Therefore, we included a range based on our determination of the information presented in the articles.

\section{Results}

We identified and screened 915 articles published between 1974 and 2016 (Figure 1). Of the 215 articles that described potential breakthrough varicella cases (publication years: 1977 to 2016), 34 met inclusion criteria for our review (publication years: 1980 to 2016): 21 articles described breakthrough varicella cases with disseminated VZV infection with other organ involvement in addition to skin; $\mathbf{9}$ articles described hospitalized breakthrough varicella cases without mention of other organ involvement in addition to skin; $\mathbf{4}$ articles described 
both [Figure 1, Table 1], for a total of 52-60 unique breakthrough varicella cases with disseminated VZV infection with other organ involvement in addition to skin and 266 hospitalized breakthrough cases without mention of other organ involvement in addition to skin. Varicella cases with disseminated VZV infection are described below; range accounts for cases we identified that potentially were reported in multiple publications (Table 2). Most (20) of the articles described severe breakthrough varicella after receipt of Merck varicella vaccine products licensed in the United States [Table 1]; this is likely due to the United States being the country with the longest duration of a routine varicella vaccination program, and with the majority of the post-licensure varicella vaccine safety articles ${ }^{20}$.

No articles indicated severe breakthrough varicella cases with disseminated VZV infection with other organ involvement in addition to skin in a person who received 2 doses of varicella vaccine. There were 2 articles that indicated hospitalized breakthrough varicella cases without mention of other organ involvement in addition to skin in 4 patients who received 2 doses of varicella vaccine ${ }^{21,22}$, although in 1 paper $^{21}$ it was unclear whether 2 of these patients were breakthrough cases or the vaccine was received $<42$ days before rash onset.

\subsection{Pneumonia}

We identified 5 articles that described breakthrough varicella cases with pneumonia. Most pneumonia cases were identified in articles describing results of post-licensure vaccine surveillance. Using data from an active surveillance project, Chaves et al ${ }^{15}$, reported 1 case of pneumonia among 1,661 breakthrough cases in children aged 1-14 years during 19972005 in the United States. Dinleyici et al ${ }^{23}$ reported 4 cases of pneumonia among a total of 36 hospitalized breakthrough cases in children from 29 health care centers in Turkey; these cases were identified as part of a national prospective study on pediatric varicella hospitalizations during 2008-2013.

Galea $^{24}$ et al reported 2 cases of pneumonia in immunocompetent persons out of 5,054 cases of breakthrough varicella based on Merck's post-marketing worldwide surveillance data 1995-2005. Quian et al ${ }^{25}$ reported 1 pneumonia case in a hospitalized 5 year-old with varicella who had been vaccinated at age 3 years among 462 varicella-related hospitalizations, of which 6 were breakthrough varicella, during 1997-2005 in Uruguay. Pileggi et al ${ }^{26}$ reported a breakthrough varicella case with pneumonia and probable macrophage activation syndrome in a patient with juvenile rheumatic disease who was on methotrexate (and possibly other immunosuppressive drugs) at the time of vaccination. The patient was a nonresponder to varicella vaccine based on serology testing using commercial assays. The patient developed severe breakthrough varicella after a known exposure to WTVZV 2-3 years after vaccination; the patient was receiving antitumor necrosis factor therapy at the time of exposure.

\subsection{Neurologic complications}

We identified 11 articles that described breakthrough cases with neurologic complications; diagnoses included meningitis, encephalitis, acute cerebellar ataxia, transverse myelitis, and cerebellitis. Seven articles described meningitis cases. Dinleyici et al $^{23}$ reported 9 
breakthrough cases with meningitis/encephalitis in their study of pediatric varicella hospitalizations; no additional information was provided on these cases. In the review of national safety data of varicella vaccine in the United States 1995-2005, Chaves et al ${ }^{10}$, described 4 cases of meningitis associated with breakthrough varicella in children aged $\unlhd 2$ years, 2 of whom had a CSF specimen positive for VZV by PCR, strain type not indicated. One of the laboratory confirmed cases was also described in a case report by Schwab et $\mathrm{al}^{27}$. This was a healthy 5 year-old who developed meningitis without ataxia who presented with fever, headache and rash. The patient had received varicella vaccine at age 3 years and developed varicella 18 months after vaccination. Upon treatment with acyclovir the patient made an uneventful recovery but at the 6 week follow-up visit, demonstrated mild to moderate sensorineural hearing loss on the right side. Two years later, the patient's hearing was normal. In their review of the safety of varicella vaccine using Merck's post-marketing worldwide surveillance database for 1995-2005, Galea et $\mathrm{al}^{24}$ reported 1 aseptic meningitis case in an immunocompetent patient with breakthrough varicella; no other details provided. Sharrar et al ${ }^{18}$ reported 1 hospitalized breakthrough varicella case with meningitis. The database used by these two publications was the same, with the Galea et al ${ }^{24}$ article covering the period analyzed by Sharrar et $\mathrm{al}^{18}$; it is likely that these were the same cases. Reynolds et $\mathrm{al}^{28}$ reported breakthrough varicella with meningitis in a 5 year-old with asthma. The child was admitted to the intensive care unit for 1 day for meningitis, and discharged with a diagnosis of altered mental status/post-varicella syndrome; by 6 weeks the child's mental status had returned to normal.

Naruse et al $^{29}$ described a 45-month old boy in Japan hospitalized with breakthrough varicella and meningitis vaccinated 21 months earlier with the Biken vaccine. After exposure to a child with varicella, the patient developed a rash (97 lesions), and headache and frequent vomiting 2 days after rash onset. The patient responded to intravenous (IV) acyclovir with resolution of symptoms within 2 days of treatment initiation and had no recurrent symptoms. His cerebrospinal fluid (CSF) specimen was positive for VZV DNA by PCR; type information not provided.

Breakthrough cases with encephalitis were described in 3 articles. One is the publication by Dinleyici et $\mathrm{al}^{23}$ that reported 9 breakthrough cases with encephalitis/meningitis. Chaves et $\mathrm{al}^{15}$, reported 1 case of breakthrough varicella with encephalitis in a healthy 4 year-old in the study from the varicella active surveillance project. Ibraheem et al ${ }^{30}$ described a 4 year-old girl without a known immunocompromising condition, who died from VZV encephalitis. Her past medical history included a nasal septum perforation with recurrent sinusitis, and hypocalcemia-related seizures at 1 year of age. She was vaccinated with 1 dose at age 13 months. The patient was admitted with sudden severe headache and right eye pain. She did not have a rash. The patient's condition worsened during hospitalization and she died on day 6 of hospitalization. PCR of post-mortem brain specimens were positive for WT-VZV DNA and VZV encephalitis was the likely cause of death ${ }^{30}$. Of note, the authors mentioned that in the absence of the rash they were unable to determine whether the patient's encephalitis was because of breakthrough infection or herpes zoster.

Acute cerebellar ataxia was described in 2 articles. Sharrar et al ${ }^{18}$ and Galea et $\mathrm{al}^{24}$ reported 2 hospitalized breakthrough varicella cases with ataxia and 2 immunocompetent case- 
patients with breakthrough varicella and ataxia, respectively. As above there is the possibility of overlap due to the use of the same database.

Two case-reports described breakthrough varicella with transverse myelitis diagnosed after the rash had resolved. LaRovere et al ${ }^{31}$ described a case in an immunocompetent 14 year-old boy, vaccinated at 6 years of age. He developed fever and a generalized papulovesicular rash that lasted 6 days. Six days after his rash resolved, the patient reported a sharp "mid-scapular pain that radiated to his chest", and 2 days later, he developed bilateral leg paresthesias and weakness, unsteady gait, and urinary retention. VZV antibody was detected in CSF. After 15 days of hospitalization, his neurologic examination was normal although he still had weakness in his extremities. Two months after presentation, he was asymptomatic and his neurologic exam was normal. Aslan et $\mathrm{al}^{32}$ described a case in a 10 year-old girl without identified immunosuppressive conditions or medications who was vaccinated at age 12 months. She was hospitalized with lower extremity paralysis and urinary retention. She had developed a mild rash (20-25 lesions) without fever, 16 days before hospitalization that lasted approximately 2 days. Fourteen days after the rash resolved, she developed bilateral leg paralysis, weakness, and unsteady gait. Her blood was positive for VZV IgG and IgM antibodies and the serum/CSF ratio of VZV antibody was reduced compared with ratios for total IgG and albumin, consistent with intrathecal synthesis of VZV antibody ${ }^{33}$. One month after hospitalization, the patient was asymptomatic and MRI showed normal findings of spinal cord.

Dinleyici et $\mathrm{al}^{23}$ reported 1 breakthrough case with cerebellitis based on hospital surveillance data from Turkey, without additional details.

\subsection{Hematologic complications}

There were 9 articles describing hematologic complications in 11 patients; diagnoses included neutropenia, thrombocytopenia, pancytopenia, possible macrophage activation syndrome, hemorrhagia, and disseminated intravascular coagulation (DIC). Dinleyici et $\mathrm{al}^{23}$ reported 4 breakthrough cases with hematologic complications: 2 with neutropenia (of which one was febrile neutropenia) and 2 with thrombocytopenia. The previously mentioned case described by Pillegi et $\mathrm{al}^{26}$ with pneumonia, also developed probable macrophage activation. Kakish et $\mathrm{al}^{34}$ described a breakthrough case in a previously healthy 4 year-old child who developed varicella 12 weeks after varicella vaccination. He had a 2-day history of fever and maculopapulovesicular rash, when he presented with ecchymotic patches on the elbows and multiple purpuric skin lesions around the eyes with bilateral subconjunctival hemorrhages. His 3 year-old sibling had recovered from varicella 10 days previously. $\mathrm{He}$ was diagnosed with pancytopenia which was not responsive to multiple treatments (IV immune globulin, platelet transfusion, pulse dose corticosteroids, granulocyte colony stimulating factor). Bone marrow aspirate showed scarcity of all cell lines. Bone marrow transplant was recommended. Follow up information was not reported.

Hemorrhagia was described in 2 articles. Chaves et al ${ }^{15}$ reported 1 breakthrough varicella case with hemorrhagia in a study using active surveillance data; no other details were provided. Reynolds et $\mathrm{al}^{28}$ reported 1 breakthrough varicella case in a previously healthy 2 
year-old who developed bleeding diathesis. These 2 papers used the same data source with overlapping years of data analyzed; it is possible that both describe the same case.

DIC was reported in 2 cases $^{18,35,36}$. These cases are described under the complication we considered initiated the chain of events that led to DIC (hepatic complications and sepsis, respectively). Lastly, Leung et al ${ }^{16}$ described a fatal breakthrough case in a 7 year-old with malignant ependymoma and on dexamethasone $(2 \mathrm{mg} /$ day) who developed thrombocytopenia during the course of her illness; this case was also reported by Galea et $\mathrm{al}^{24}$. Limited clinical information was available on this case.

\subsection{Ocular complications}

There were 5 articles that described 5 breakthrough varicella patients with ocular complications, which included keratitis, conjunctivitis, subconjunctival hemorrhage, retinitis, blurred vision, and hemorrhagic retinitis. Galea et al ${ }^{24}$ reported 1 patient with breakthrough varicella and keratitis. Kurugol et al ${ }^{37}$ reported conjunctivitis in a 10 year-old healthy child from Turkey who developed breakthrough 9 years after receipt of 1-dose of varicella vaccine. The child was hospitalized for disseminated rash and fever and received oral and topical acyclovir for conjunctivitis; the patient fully recovered. The patient described above by Kakish et al ${ }^{34}$ with hematologic complications, also developed ocular complications (bilateral subconjunctival hemorrhages).

Ross et $\mathrm{al}^{38}$ described 1 breakthrough case in a 7 year-old child with T-cell acute lymphoblastic leukemia (ALL) in remission who developed varicella retinitis, blurred vision, and hemorrhagic retinitis. He was vaccinated with 1 dose at age 4, 27 months before he was diagnosed with ALL at age 6 years. He was VZV IgG positive at the time of his ALL diagnosis. He presented to the oncology clinic with high fever $\left(104^{\circ} \mathrm{F}\right)$ and generalized vesicular rash and was clinically diagnosed with varicella. His last course of chemotherapy was 3 weeks before rash onset. He was exposed to a child with varicella in his school. After IV and oral acyclovir the rash resolved and the patient was discharged with all skin lesions crusted. Two weeks following varicella, he presented with blurred vision in the right eye, without new skin lesions. He developed a right afferent pupillary defect and superior temporal defect, and subsequently hemorrhagic retinitis in the inferotemporal quadrant. $\mathrm{He}$ was re-admitted, and received prolonged therapy with acyclovir and prednisolone ophthalmic drops that prevented progression of the retinitis. A residual scar remained in the area of previous retinitis.

Additionally, Dinleyici et al ${ }^{23}$ identified 1 hospitalized breakthrough varicella case with severe eye involvement; details of the ocular complications were not included.

\subsection{Renal complications}

There were 2 articles that described renal complications in 2 patients. Pillai et al ${ }^{39}$ described a breakthrough case in a 24 year-old male with no history of renal disease or varicella who was vaccinated almost 2 years prior. He presented to the hospital with rash, gross hematuria, shaking chills, fever, and mild hypertension (130/94). Urinalysis revealed 4+ protein, 4+ blood, many red blood cells (RBC), and granular casts. Blood pressure and 
urinary abnormalities resolved in approximately 3 weeks; urinalysis 7 months later revealed 3-5 RBCs. WT-VZV was cultured from skin lesion specimens. Goulleret et $\mathrm{al}^{11}$ reported 1 case with renal complications out of 261 cases of breakthrough varicella based on Merck's post-licensure surveillance data from Europe during 2003-2008. The case occurred in a 3 year-old who was vaccinated at age 12 months, and 2 years later developed breakthrough varicella with nephrotic syndrome that required hospitalization. The child recovered completely. No specimens were collected for VZV laboratory testing.

\subsection{Hepatic complications}

There were 4 articles that described 3 cases of breakthrough varicella with hepatic complications including hepatomegaly, hepatitis, and hepatic necrosis. Nunoue et al ${ }^{40}$ described 1 case with hepatomegaly. This was a child with acute leukemia treated with immunosuppressive therapy at the time of vaccination and after vaccination. She was vaccinated at age 3 years and developed varicella 11 months after vaccination. Her symptoms included fever, generalized rash (>100 lesions) that lasted 10 days, hepatomegaly, and elevated amino transferase levels. VZV culture was negative.

Kelley et al ${ }^{41}$ described 1 case with hepatitis that resulted in multi-organ failure and death; this death was also included in the summary of breakthrough varicella deaths in the paper by Leung et $\mathrm{al}^{16}$. This was a breakthrough case in a 15 year-old boy with T-cell precursor ALL who was undergoing reinduction chemotherapy and on Decadron $(20 \mathrm{mg} /$ day $)$. He had received 1-dose of varicella vaccine at age 2 years (based on our earlier article ${ }^{16}$; Kelley et $\mathrm{al}^{41}$ reported he was vaccinated at age 5), and was up-to-date on other vaccinations. He presented with fever and a sharp stabbing back pain and 3 days later developed a rash described as nonvesiculated acne-like believed to be related to medication; on day 7 the rash started to vesiculate and PCR identified WT-VZV DNA in the vesicular fluid. On day 9 of illness, the patient developed hepatitis, and subsequently multi-organ failure and died. Wise et $\mathrm{al}^{36}$ and Sharrar et al ${ }^{18}$ each describe the same fatal breakthrough varicella case in a 9 year-old girl with chronic severe asthma, on high-dose inhaled corticosteroids (60 mg/day, steroid not specified). This patient had received the $1^{\text {st }}$ dose of varicella vaccine shortly before her $8^{\text {th }}$ birthday. No rash had been recognized before death, but at autopsy, 2 of several scattered papules showed "intraepidermal vesicles with viral inclusions and multinucleated cells". Autopsy disclosed fulminant hepatitis, esophagitis and epiglottitis, DIC and positive VZV cultures from pharynx, lung, blood, and urine; PCR identified WTVZV. Her liver was diffusely necrotic, and few surviving hepatocytes showed viral cytopathic changes.

\subsection{Sepsis and Secondary Infection resulting in bacteremia}

There were 7 articles that described sepsis (bacterial or viral) as a complication in 8 breakthrough varicella cases. The specific organism that caused sepsis was reported in 3 cases (Staph aureus or Group A streptococcus) ${ }^{18,35,36,42}$. The first case was a fatal case of breakthrough varicella described by Sharrar et al ${ }^{18}$ and Wise et al ${ }^{36}$, mentioned above with hepatic complications that also developed Staph aureus sepsis. The second case, described by Kwak et $\mathrm{al}^{35}$, was in a previously healthy 38 month-old girl with necrotizing fasciitis on the left abdominal wall and streptococcal toxic shock syndrome; she was vaccinated at 12 
months. Three days after onset of rash, she was seen in the emergency department for increased pain and swelling in the left flank. She had several ruptured blisters, macular rash, and painful purplish swelling surrounding some of the skin lesions on her left flank. Computerized tomography scan showed extensive soft tissue swelling of the left anterior abdominal wall, both flanks, and inguinal area and magnetic resonance imaging identified necrotizing fasciitis on the same sites. Group A Streptococcus was isolated by tissue culture. Laboratory investigation revealed disseminated intravascular coagulation. The patient recovered and was discharged after 5 weeks of hospitalization. The third case, described by Laskey et $\mathrm{al}^{42}$, was in a previously healthy 3 year-old boy who developed varicella 6 months after vaccination with no apparent skin infection, but the patient developed group A $\beta$ hemolytic streptococcus (GABHS) bacteremia, resulting in endocarditis. Ten days after rash onset, the patient was admitted with a 3-day history of nausea, vomiting, fever along with increased irritability, decreased urine output and right foot pain with difficulty walking. Examination revealed crusted skin lesions without erythema, an I/VI systolic ejection murmur and worsening joint pain. On hospital day 2 blood cultures were positive for GABHS. The hospital course was complicated by bilateral pulmonary infiltrates, hypoalbuminemia, elevated liver function tests. On hospital day 8, the patient developed a diastolic murmur consistent with aortic regurgitation and subungal hemorrhages were noted. Echocardiography revealed thickening of the aortic valve with moderate aortic insufficiency. The patient was started on a 6-week treatment with IV penicillin G. However, after 2 weeks, the diastolic murmur was harsher and echocardiography revealed a large vegetation on the posterior leaflet of the aortic valve with severe aortic insufficiency and a dilated left ventricle. He was treated for congestive heart failure. A month after the initial echocardiography, the patient continued to have worsening aortic regurgitation and mitral regurgitation. The patient received an additional 4 weeks of antibiotic followed by aortic valve replacement. He had an unremarkable postoperative course and was doing well on follow-up examination.

There were 5 additional cases of sepsis reported, without information on the specific organism leading to sepsis, 3 alleged to be cases of bacterial sepsis. Dinleyici et al ${ }^{23}$ identified 1 breakthrough varicella case in a child with leukemia who developed a secondary bacterial infection and sepsis, who ultimately died. Galea et $\mathrm{al}^{24}$ reported 2 breakthrough cases in immunocompetent persons who developed sepsis from secondary infection. The 7 year-old patient with malignant ependymoma, described earlier with thrombocytopenia and reported by Galea et $\mathrm{al}^{24}$ and Leung et $\mathrm{a}^{16}$, also developed sepsis. This case had no evidence of bacterial infection (unpublished surveillance data). Sharrar et al ${ }^{18}$ reported an additional hospitalized breakthrough varicella case with dehydration and possible sepsis.

\subsection{Other complications}

Other complications included hypotension and transient hearing loss, which were described in the 2 cases mentioned above, reported by Kwak et $\mathrm{al}^{35}$ (described under sepsis) and Schwab et al ${ }^{27}$ (described under neurologic complications), respectively. Galea et al ${ }^{24}$ reported 1 patient with breakthrough varicella who developed myocarditis; no other details were provided on this case. Kriner et $\mathrm{al}^{43}$ described 1 fatal breakthrough case in a patient 
with ALL who developed multi-organ failure; she was on maintenance therapy (Vincristine/ dexamethasone).

\subsection{Other Hospitalizations}

Thirteen articles described a hospitalized breakthrough varicella case without mention of other organ involvement in addition to skin for a total estimated number of 266 cases $^{18,21-23,25,28,44-50}$. Eleven of these articles described data from various surveillance systems in different countries $18,21-23,25,28,44,45,47,49,50$. Among 8 with information on total number of hospitalized varicella cases, in 7 the proportion of breakthrough hospitalizations among all varicella hospitalizations was $0-4 \%$; in 1 the proportion was $12 \% 45$. From 7 papers with information on the interval between varicella vaccination and hospitalization, the median interval was $0.8-4.7$ years (range, $0.1-14$ years) $18,22,23,25,28,45,50$. Where information was provided, majority of these hospitalized cases occurred in immunocompromised children or adolescents and reasons for hospitalization included cellulitis, secondary bacterial infection limited to the skin, dehydration, or for precautionary reasons due to their immune status. Additionally, Yamada et al ${ }^{46}$ described 1 breakthrough varicella case in a 12 year-old cancer patient who was vaccinated at age 1 . The patient was hospitalized for a prolonged rash for at least 14 days, with a maximum of 12 lesions at the peak of illness. Haddad et al ${ }^{48}$, described a varicella outbreak in a school, which involved one student with severe rash with $>500$ lesions who was hospitalized for 3 days and treated with IV antibiotics for Group A streptococcal secondary skin infection. Hospitalized breakthrough varicella with febrile seizure was reported by Dinleyici et $\mathrm{al}^{23}$ in Turkey ( 2 cases), Streng et al ${ }^{49}$ in Germany ( 2 cases) and Quian et $\mathrm{al}^{25}$ in Uruguay ( 1 case in a 2 year-old vaccinated 4 months previously).

\subsection{Death}

There have been 6 cases of fatal breakthrough varicella reported in 8 articles, all described above $^{16,18,23,24,30,36,41,43}$. Five of these deaths were reported from the United States and summarized in the article by Leung et $\mathrm{al}^{16}$, the $6^{\text {th }}$ death was reported from Turkey by Dinleyici et $\mathrm{al}^{23}$. The deaths occurred in 1-dose vaccinees (interval between vaccination and death was 1-13 years; information available for 5 cases), in children aged $<18$ years (ages 4 , $4,7,9,15$ and $<18$ years) and 5 of the 6 occurred in immunocompromised persons due to either medical conditions or medications; the year of death was 1997, 2004, 2011, 2012, 2013, and 2008-2013 for the death reported from Turkey (specific year of death not indicated in the publication). Reported complications included: hepatitis, hemorrhagia, and sepsis $^{18,36}$; sepsis and thrombocytopenia ${ }^{16,24}$; hepatitis and multi-organ failure ${ }^{41}$; sepsis ${ }^{23}$; encephalitis ${ }^{30}$; and multi-organ failure ${ }^{43}$.

\section{Expert Commentary and five-year view}

Varicella vaccines have led to substantial declines in varicella disease $3,9,10,12,51$. The World Health Organization estimated an annual average of 31 million varicella vaccine doses distributed worldwide between 2007 and 201152; in the US >145 million doses of varicella vaccine have been distributed since program implementation through 2013. No vaccine is $100 \%$ safe or effective and adverse events following vaccination, and cases of 
vaccine failure, including breakthrough varicella, have been reported. A review of the epidemiological and mechanistic (clinical and biological) evidence of selected adverse events after varicella vaccination associated with Oka VZV (i.e., vaccine-strain) was performed by the Institute of Medicine (IOM) in $2011^{20}$. We focused our review on the occurrence of varicella due to wild-type VZV in previously vaccinated persons (i.e., vaccine failure), which was not reviewed by the IOM report.

This is the first systematic review to describe clinical characteristics and complications of severe varicella presentations among vaccinated children and summarize the information reported in the literature. Considering primarily the number of skin lesions, previous studies of varicella among vaccinated children described cases of breakthrough as mostly mild but indicate that $25 \%$ of cases had clinical presentation more similar to unvaccinated children ${ }^{15}$. Most of these studies were not large enough to detect uncommon, serious complications of breakthrough varicella. Chaves et $\mathrm{al}^{53}$, reported that vaccinated children with $\geq 50$ lesions were twice as likely to have complications, such as pneumonia, ataxia and skin superinfections, than vaccinated children with $<50$ lesions. Our review documented that severe complications with disseminated VZV infection with other organ involvement in addition to skin among breakthrough cases do occur but they appear uncommon. We found a similar pattern of complications in breakthrough varicella as compared to varicella in unvaccinated children with pneumonia, neurologic complications, and bacterial superinfection being most common ${ }^{2}$. Based on the number of published case-reports and cases identified by the post-marketing surveillance, severe complications of breakthrough varicella are likely less common than severe complications among unvaccinated patients. Ecologically, this statement is supported by evidence from studies that documented substantial declines in severe outcomes of varicella (i.e., hospitalizations and deaths) in countries with a varicella vaccination program $16,22,49,51,54,55$. Even in the presence of a decline, when information on vaccination status was known, studies documented that the majority of hospitalizations which continue to occur are among unvaccinated patients.

Besides describing the types of complications and likely rarity of severe breakthrough varicella, our review allows for several other conclusions. First, although more frequent among immunocompromised children, severe breakthrough varicella-related outcomes can occur among healthy children too. Second, the data do not suggest that severe breakthrough varicella are more likely to occur with increased time since vaccination as most published cases occurred in children, rather than in young adults. Although we were not able to formally evaluate this question because of the lack of information on all cases, we found that majority of severe breakthrough cases that resulted in disseminated VZV infection with other organ involvement in addition to skin occurred within 5 years of varicella vaccination with 4 cases that occurred $8-13$ years post-vaccination ${ }^{16,31,32,37,41}$; hospitalizations of breakthrough varicella without mention of other organ involvement in addition to skin occurred at a median of $0.8-4.7$ years post-vaccination $18,22,23,25,28,45,50$. The question of whether severe breakthrough varicella is related to time since vaccination will be important to continue monitoring. Third, we did not identify any severe complications with disseminated VZV infection with other organ involvement in addition to skin among 2 dose vaccine recipients; there were 4 hospitalized breakthrough cases with skin infection who received 2 doses of varicella vaccine. Nonetheless, physicians should consider in the 
differential diagnosis possible varicella-related complications when biologically plausible, even when varicella vaccine was received. Additionally, given the atypical presentations of breakthrough varicella, a diagnosis of varicella in vaccinated persons may not be readily made or a mild rash with few lesions may be missed altogether. Continued surveillance for varicella disease among vaccinated persons is important to evaluate the varicella vaccination program, detect any changes in the epidemiology of varicella in the context of a routine oneor two-dose varicella vaccination program, and understand the differences in disease presentation between persons who had received 1 or 2 doses of varicella vaccine.

Our review has several limitations. We were unable to calculate rates of complications for severe breakthrough varicella. It was challenging to determine the number of cases in each symptom category group since a case may have been reported in multiple articles. We indicated the potential overlap in reporting of the same breakthrough cases among different articles when information in the articles allowed us to make that determination. There was limited information on clinical course, medical history, and vaccination history for some of the cases with information often presented as aggregate data instead of case-level data. We may have missed severe breakthrough cases because of under-reporting; however, we believe that these cases would be more likely to be reported. Our literature review does not include data on all hospitalized breakthrough cases and therefore the number of hospitalizations we report should be interpreted with this caveat. The 12 articles included in our review described postlicensure surveillance from 9 countries and may have only included data on select populations or surveillance sites. Additionally, data from national datasets in the United States are not included because information on vaccination status is not available in these datasets. Nonetheless, in the articles included in our review, the proportion of breakthrough varicella cases among all varicella-related hospitalizations is small, ranging from 0 to $4 \%$.

The majority of cases were clinically diagnosed. Confirmation as WT-VZV infection was limited (5 cases, with additional 3 cases with VZV DNA detected but not typed). Even for cases with laboratory-confirmed WT-VZV infection, we are unable to definitively link the complications to WT-VZV infection. Some of the complications could have also been caused by vaccine-strain VZV during primary infection or re-activation since the vaccinestrain is a live virus. However, we minimized these possibilities by 1) excluding varicellalike cases that occurred $<42$ days post-vaccination, which are more likely to represent vaccine-strain varicella cases, and 2) excluding cases diagnosed as herpes zoster or with localized rash suggestive of herpes zoster. Lastly, we excluded cases from one article that analyzed data from postlicensure surveillance systems in which severe outcomes were reported at varying intervals (6-128 days) after vaccination among persons who received varicella vaccine and that occurred in the absence of rash, a diagnosis of breakthrough varicella, or laboratory confirmation of VZV infection ${ }^{36}$; it is unlikely that these cases were associated with breakthrough varicella.

\section{Acknowledgments}

Funding Details: None 


\section{References}

1. Gershon, A., Takahashi, M., Seward, JF. Varicella vaccine. In: Plotkin, S.Orenstein, W., Offit, P., editors. Vaccines. 5. Saunders: Elsevier; 2008. p. 915-958.

2. Galil K, Brown C, Lin F, Seward J. Hospitalizations for varicella in the United States, 1988 to 1999. The Pediatric infectious disease journal. 2002; 21:931-935. [PubMed: 12394815]

3. Marin M, Guris D, Chaves SS, Schmid S, Seward JF. Prevention of varicella: recommendations of the Advisory Committee on Immunization Practices (ACIP). MMWR Recommendations and reports : Morbidity and mortality weekly report Recommendations and reports / Centers for Disease Control. 2007; 56:1-40.

4. Takahashi M, Otsuka T, Okuno Y, Asano Y, Yazaki T. Live vaccine used to prevent the spread of varicella in children in hospital. Lancet. 1974; 2:1288-1290. [PubMed: 4139526]

5. Kim JI, Jung GS, Kim YY, Ji GY, Kim HS, Wang WD, et al. Sequencing and characterization of Varicella-zoster virus vaccine strain SuduVax. Virology journal. 2011; 8:547. [PubMed: 22176950]

6. WHO. [Accessed 4 Feb 2016] Varicella Vaccine. WHO vaccine-preventable diseases: monitoring system. 2015 global summary. Available at http://apps.who.int/immunization_monitoring/ globalsummary/schedules?sc $\% 5 \mathrm{Br} \% 5 \mathrm{D} \% 5 \mathrm{~B} \% 5 \mathrm{D}=\mathrm{AFRO} \& \mathrm{sc} \% 5 \mathrm{Br} \% 5 \mathrm{D} \% 5 \mathrm{~B} \% 5 \mathrm{D}=\mathrm{AMRO} \& \mathrm{sc}$ $\% 5 \mathrm{Br} \% 5 \mathrm{D} \% 5 \mathrm{~B} \% 5 \mathrm{D}=\mathrm{EMRO} \& \mathrm{sc} \% 5 \mathrm{Br} \% 5 \mathrm{D} \% 5 \mathrm{~B} \% 5 \mathrm{D}=\mathrm{EURO} \& \mathrm{sc} \% 5 \mathrm{Br} \% 5 \mathrm{D} \% 5 \mathrm{~B}$ $\% 5 \mathrm{D}=\mathrm{SEARO} \& \mathrm{sc} \% 5 \mathrm{Br} \% 5 \mathrm{D} \% 5 \mathrm{~B} \% 5 \mathrm{D}=\mathrm{WPRO} \& \mathrm{sc} \% 5 \mathrm{Bd} \% 5 \mathrm{D}=\& \mathrm{sc} \% 5 \mathrm{Bv} \% 5 \mathrm{D} \% 5 \mathrm{~B}$ $\% 5 \mathrm{D}=$ VARICELLA\&sc $\% 5 \mathrm{BOK} \% 5 \mathrm{D}=\mathrm{OK}$

7. Varicella and herpes zoster vaccines: WHO position paper, June 2014. Releve epidemiologique hebdomadaire / Section d'hygiene du Secretariat de la Societe des Nations = Weekly epidemiological record / Health Section of the Secretariat of the League of Nations. 2014; 89:265287.

8. Organization/UNICEF. WH. Immunization summary: the 2014 edition. Geneva: World Health Organization; 2013. Available at http://www.childinfo.org/files/ immunization_summary_2012_en.pdf [Accessed 22 February 2016]

9. Marin MMM, Kambhampati A, Jeram SM, Seward JF. Global Varicella Vaccine Effectiveness: A Meta-analysis. Pediatrics published online. Feb 16.2016 doi:10.1542.2016.

10. Chaves SS, Haber P, Walton K, Wise RP, Izurieta HS, Schmid DS, et al. Safety of varicella vaccine after licensure in the United States: experience from reports to the vaccine adverse event reporting system, 1995-2005. The Journal of infectious diseases. 2008; 197(Suppl 2):S170-177. [PubMed: 18419393]

11. Goulleret N, Mauvisseau E, Essevaz-Roulet M, Quinlivan M, Breuer J. Safety profile of live varicella virus vaccine (Oka/Merck): five-year results of the European Varicella Zoster Virus Identification Program (EU VZVIP). Vaccine. 2010; 28:5878-5882. [PubMed: 20600487]

12. Kreth HW, Lee BW, Kosuwon P, Salazar J, Gloriani-Barzaga N, Bock HL, et al. Sixteen years of global experience with the first refrigerator-stable varicella vaccine (Varilrix). BioDrugs : clinical immunotherapeutics, biopharmaceuticals and gene therapy. 2008; 22:387-402.

13. Shapiro ED, Vazquez M, Esposito D, Holabird N, Steinberg SP, Dziura J, et al. Effectiveness of 2 doses of varicella vaccine in children. The Journal of infectious diseases. 2011; 203:312-315. [PubMed: 21208922]

14. Thomas CA, Shwe T, Bixler D, del Rosario M, Grytdal S, Wang C, et al. Two-dose varicella vaccine effectiveness and rash severity in outbreaks of varicella among public school students. The Pediatric infectious disease journal. 2014; 33:1164-1168. [PubMed: 24911894]

15. Chaves SS, Zhang J, Civen R, Watson BM, Carbajal T, Perella D, et al. Varicella disease among vaccinated persons: clinical and epidemiological characteristics, 1997-2005. The Journal of infectious diseases. 2008; 197(Suppl 2):S127-131. [PubMed: 18419385]

16. Leung J, Bialek SR, Marin M. Trends in varicella mortality in the United States: Data from vital statistics and the national surveillance system. Human vaccines \& immunotherapeutics. 2015; 11:662-668. [PubMed: 25714052]

17. [Accessed 15 Dec 2015] Varicella: Chapter 17-1. VPD Surveillance Manual. 52011. Available at: http://www.cdc.gov/vaccines/pubs/surv-manual/chpt17-varicella.pdf. Available 
18. Sharrar RG, LaRussa P, Galea SA, Steinberg SP, Sweet AR, Keatley RM, et al. The postmarketing safety profile of varicella vaccine. Vaccine. 2000; 19:916-923. [PubMed: 11115716]

19. Harpaz R, Ortega-Sanchez IR, Seward JF. Prevention of herpes zoster: recommendations of the Advisory Committee on Immunization Practices (ACIP). MMWR Recommendations and reports : Morbidity and mortality weekly report Recommendations and reports / Centers for Disease Control. 2008; 57:1-30. quiz CE32-34.

20. Stratton, K.Ford, A.Rusch, E., Clayton, EW., editors. Adverse Effects of Vaccines: Evidence and Causality. Washington DC: 2012 by the National Academy of Sciences; 2011. Varicella Virus Vaccine.

21. Agopian A, Lopez A, Wilson D, Peralta V, El Amin AN, Bialek S. Varicella hospitalizations in Los Angeles during the varicella vaccination era, 2003-2011: are they preventable? Vaccine. 2014; 32:5353-5356. [PubMed: 25087675]

22. Tan B, Bettinger J, McConnell A, Scheifele D, Halperin S, Vaudry W, et al. The effect of funded varicella immunization programs on varicella-related hospitalizations in IMPACT centers, Canada, 2000-2008. The Pediatric infectious disease journal. 2012; 31:956-963. [PubMed: 22647896]

23. Dinleyici EC, Kurugol Z, Kara A, Tezer H, Tas MA, Guler E, et al. Children with breakthrough varicella infection requiring hospitalization in Turkey (VARICOMP Study 2008-2013). Vaccine. 2015; 33:3983-3987. [PubMed: 26133048]

24. Galea SA, Sweet A, Beninger P, Steinberg SP, Larussa PS, Gershon AA, et al. The safety profile of varicella vaccine: a 10-year review. The Journal of infectious diseases. 2008; 197(Suppl 2):S165169. [PubMed: 18419392]

25. Quian J, Ruttimann R, Romero C, Dall' Orso P, Cerisola A, Breuer T, et al. Impact of universal varicella vaccination on 1-year-olds in Uruguay: 1997-2005. Archives of disease in childhood. 2008; 93:845-850. [PubMed: 18456699]

26. Pileggi GS, de Souza CB, Ferriani VP. Safety and immunogenicity of varicella vaccine in patients with juvenile rheumatic diseases receiving methotrexate and corticosteroids. Arthritis care \& research. 2010; 62:1034-1039. [PubMed: 20235203]

27. Schwab J, Ryan M. Varicella zoster virus meningitis in a previously immunized child. Pediatrics. 2004; 114:e273-274. [PubMed: 15286270]

28. Reynolds MA, Watson BM, Plott-Adams KK, Jumaan AO, Galil K, Maupin TJ, et al. Epidemiology of varicella hospitalizations in the United States, 1995-2005. The Journal of infectious diseases. 2008; 197(Suppl 2):S120-126. [PubMed: 18419384]

29. Naruse H, Miwata H, Ozaki T, Asano Y, Namazue J, Yamanishi K. Varicella infection complicated with meningitis after immunization. Acta paediatrica Japonica; Overseas edition. 1993; 35:345_ 347. [PubMed: 8397466]

30. Ibraheem M, Marin M, Leung J, Bryce CH, Schmid DS, Zaki SR, et al. Fatal wild-type varicellazoster virus encephalitis without a rash in a vaccinated child. The Pediatric infectious disease journal. 2013; 32:183-185. [PubMed: 22982982]

31. LaRovere KL, Raju GP, Gorman MP. Postvaricella acute transverse myelitis in a previously vaccinated child. Pediatric neurology. 2008; 38:370-372. [PubMed: 18410857]

32. Aslan A, Kurugol Z, Gokben S. Acute transverse myelitis complicating breakthrough varicella infection. The Pediatric infectious disease journal. 2014; 33:1196-1198. [PubMed: 24853543]

33. Birlea M, Arendt G, Orhan E, Schmid DS, Bellini WJ, Schmidt C, et al. Subclinical reactivation of varicella zoster virus in all stages of HIV infection. Journal of the neurological sciences. 2011; 304:22-24. [PubMed: 21419427]

34. Kakish KBR, Dhuhouri JA, Chakraborty S. Four Year Old Child with Breakthrough Varicella Leading to Pancytopenia. Bahrain Medical Bulletin. 2009; 31:1-4.

35. Kwak BO, Lee MJ, Park HW, Song MK, Chung S, Kim KS. Necrotizing fasciitis and streptococcal toxic shock syndrome secondary to varicella in a healthy child. Korean journal of pediatrics. 2014; 57:538-541. [PubMed: 25653688]

36. Wise RP, Salive ME, Braun MM, Mootrey GT, Seward JF, Rider LG, et al. Postlicensure safety surveillance for varicella vaccine. JAMA : the journal of the American Medical Association. 2000; 284:1271-1279. [PubMed: 10979114] 
37. Kurugol Z, Halicioglu O, Koc F, Koturoglu G, Aksit S. Varicella rates among unvaccinated and one-dose vaccinated healthy children in Izmir, Turkey. International journal of infectious diseases : IJID : official publication of the International Society for Infectious Diseases. 2011; 15:e475-480. [PubMed: 21592838]

38. Ross A, McLean TW, Farber R, Weaver RG Jr, Chauvenet A, Givner LB, et al. Retinitis following varicella in a vaccinated child with acute lymphoblastic leukemia. Pediatric blood \& cancer. 2005; 45:191-194. [PubMed: 15880424]

39. Pillai JJ, Gaughan WJ, Watson B, Sivalingam JJ, Murphey SA. Renal involvement in association with postvaccination varicella. Clinical infectious diseases : an official publication of the Infectious Diseases Society of America. 1993; 17:1079-1080. [PubMed: 8110946]

40. Nunoue T. Clinical observations on varicella-zoster vaccinees treated with immunosuppressants for a malignancy. Biken journal. 1984; 27:115-118. [PubMed: 6100047]

41. Kelley J, Tristram D, Yamada M, Grose C. Failure of a Single Varicella Vaccination to Protect Children With Cancer From Life-Threatening Breakthrough Varicella. The Pediatric infectious disease journal. 2015; 34:1027-1029. [PubMed: 25955833]

42. Laskey AL, Johnson TR, Dagartzikas MI, Tobias JD. Endocarditis attributable to group A betahemolytic streptococcus after uncomplicated varicella in a vaccinated child. Pediatrics. 2000; 106:E40. [PubMed: 10969124]

43. Kriner P, Lopez K, Leung J, Harpaz R, Bialek SR. Notes from the field: varicella-associated death of a vaccinated child with leukemia - California, 2012. MMWR Morbidity and mortality weekly report. 2014; 63:161. [PubMed: 24553201]

44. Lerner-Geva L, Hirsh-Yechezkel G, Novikov I, Farkash H, Boyko V, Spirer Z, et al. The effect of active immunization on varicella-related hospitalizations in Israel. Human vaccines. 2009; 5:136140. [PubMed: 19246993]

45. Marshall HS, McIntyre P, Richmond P, Buttery JP, Royle JA, Gold MS, et al. Changes in patterns of hospitalized children with varicella and of associated varicella genotypes after introduction of varicella vaccine in Australia. The Pediatric infectious disease journal. 2013; 32:530-537. [PubMed: 23249914]

46. Yamada M, Kamberos N, Grose C. Breakthrough varicella in a cancer patient with persistent varicella antibody after one varicella vaccination. The Journal of pediatrics. 2013; 163:1511-1513. [PubMed: 23932212]

47. Zurynski Y, McIntyre P, Booy R, Elliott EJ. Paediatric active enhanced disease surveillance: a new surveillance system for Australia. Journal of paediatrics and child health. 2013; 49:588-594. [PubMed: 23781900]

48. Haddad MB, Hill MB, Pavia AT, Green CE, Jumaan AO, De AK, et al. Vaccine effectiveness during a varicella outbreak among schoolchildren: Utah, 2002-2003. Pediatrics. 2005; 115:14881493. [PubMed: 15930208]

49. Streng A, Grote V, Carr D, Hagemann C, Liese JG. Varicella routine vaccination and the effects on varicella epidemiology - results from the Bavarian Varicella Surveillance Project (BaVariPro), 2006-2011. BMC infectious diseases. 2013; 13:303. [PubMed: 23815523]

50. Huang WC, Huang LM, Chang IS, Tsai FY, Chang LY. Varicella breakthrough infection and vaccine effectiveness in Taiwan. Vaccine. 2011; 29:2756-2760. [PubMed: 21315697]

51. Bialek SR, Perella D, Zhang J, Mascola L, Viner K, Jackson C, et al. Impact of a routine two-dose varicella vaccination program on varicella epidemiology. Pediatrics. 2013; 132:e1134-1140. [PubMed: 24101763]

52. World Health Organization. [last accessed April 20, 2016] SAGE working group on varicella and herpes zoster vaccines. Background paper on varicella vaccine. Available at http://www.who.int/ immunization/sage/meetings/2014/april/1_SAGE_varicella_background_paper_FINAL.pdf

53. Chaves SS, Gargiullo P, Zhang JX, Civen R, Guris D, Mascola L, et al. Loss of vaccine-induced immunity to varicella over time. The New England journal of medicine. 2007; 356:1121-1129. [PubMed: 17360990]

54. Siedler A, Dettmann M. Hospitalization with varicella and shingles before and after introduction of childhood varicella vaccination in Germany. Human vaccines \& immunotherapeutics. 2014; 10:3594-3600. [PubMed: 25483695] 
55. Leung J, Harpaz R. Impact of the Maturing Varicella Vaccination Program on Varicella and Related Outcomes in the United States: 1994-2012. Journal of the Pediatric Infectious Diseases Society. 2015

56. LaRussa, PS., Marin, M. Varicella-Zoster Virus. In: Kliegman, RM.Stanton, BF.St Geme, JW., Schor, NF., editors. Nelson Textbook of Pediatrics. 20. Philadelphia, PA: Elsevier; 2016. p. $1579-1586$ 


\section{Key Issues}

\section{Main messages}

- $\quad$ Severe varicella in vaccinated persons (breakthrough varicella) involving disseminated VZV infection with other organ involvement in addition to skin, other hospitalizations, or death can occur but they appear to be uncommon. Providers should consider varicella as part of the differential diagnosis of severe clinical presentations when biologically plausible, even if a person had received varicella vaccine; rapid antiviral treatment is important in these situations ${ }^{56}$.

- With $>31$ million varicella vaccine doses distributed annually worldwide since 2007, 34 published articles described severe breakthrough varicella; of them 25 described $\sim 60$ cases with disseminated VZV infection with other organ involvement in addition to skin. Complications of varicella are likely less common in persons who were vaccinated than those who were not vaccinated.

- Complications involving disseminated VZV infection with other organ involvement in addition to skin in persons with breakthrough varicella are similar to those seen in varicella among unvaccinated persons. These complications were reported among one-dose vaccine recipients only; none were reported among two-dose vaccine recipients.

- Of hospitalized breakthrough varicella cases without mention of other organ involvement in addition to skin, a few (4) were reported in two-dose vaccine recipients, but the majority were reported among one-dose vaccine recipients.

- Severe breakthrough varicella can occur among healthy persons, but appear to be more common among immunocompromised persons, who are usually not recommended to receive varicella vaccination.

- Varicella vaccines are safe and effective, and had a significant impact on reducing varicella morbidity and mortality.

- $\quad$ Continued surveillance for varicella disease among vaccinated persons is important to determine changes in the varicella epidemiology and whether changes in disease presentation with time since vaccination occur. 


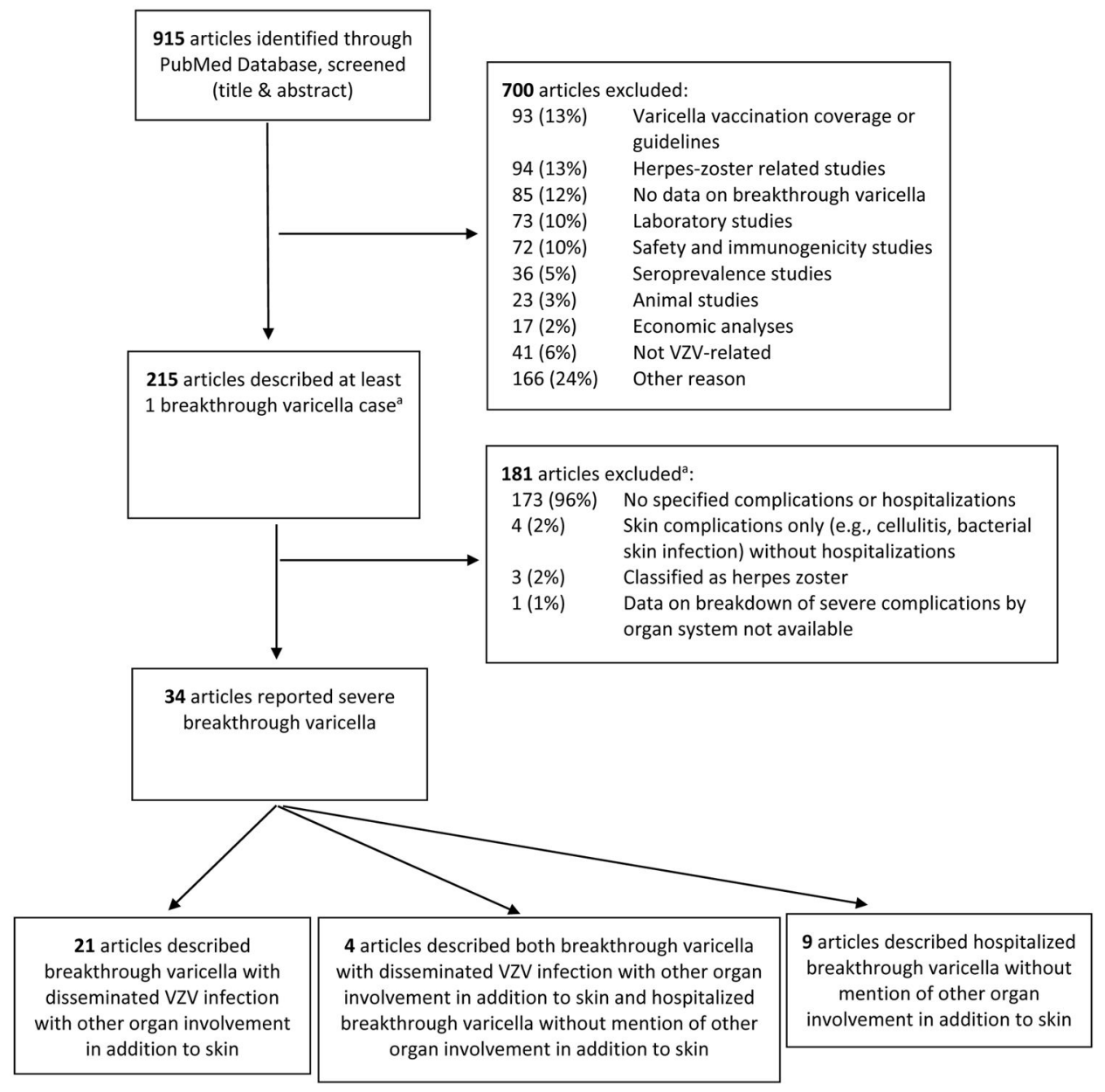

Figure 1.

Article selection

a 17 articles not available in English and only abstract reviewed. Abstracts did not indicate varicella complications or hospitalizations (15 articles) or reported cases were classified as herpes zoster ( 2 articles)

Expert Rev Vaccines. Author manuscript; available in PMC 2017 August 04. 
Table 1

Overview of 34 References Describing Severe Breakthrough Varicella

\begin{tabular}{|c|c|}
\hline Variable Category & \# Articles (\%) \\
\hline \multicolumn{2}{|l|}{ Article Publication Year } \\
\hline $1980-1994^{a}$ & $3(9 \%)$ \\
\hline 1995-2009 & $14(41 \%)$ \\
\hline 2010-2016 & $17(50 \%)$ \\
\hline \multicolumn{2}{|l|}{ Country } \\
\hline United States & $18(53 \%)$ \\
\hline Turkey & $3(9 \%)$ \\
\hline Australia & $2(6 \%)$ \\
\hline Japan & $2(6 \%)$ \\
\hline Europe $^{b}$ & $2(6 \%)$ \\
\hline Brazil & $1(3 \%)$ \\
\hline Canada & $1(3 \%)$ \\
\hline Israel & $1(3 \%)$ \\
\hline Korea & $1(3 \%)$ \\
\hline Taiwan & $1(3 \%)$ \\
\hline United Arab Emirates & $1(3 \%)$ \\
\hline Uruguay & $1(3 \%)$ \\
\hline \multicolumn{2}{|l|}{ Article Type } \\
\hline Post-licensure disease surveillance & $13(38 \%)$ \\
\hline Case-report & $13(38 \%)$ \\
\hline Post-licensure safety and/or immunogenicity report & $6(18 \%)$ \\
\hline Pre-licensure safety and efficacy & $1(3 \%)$ \\
\hline Outbreak investigation report & $1(3 \%)$ \\
\hline \multicolumn{2}{|l|}{ Number of vaccine doses among patients described } \\
\hline 1 Dose & $22(65 \%)$ \\
\hline$\geq 1$ Doses $^{c}$ & $2(6 \%)$ \\
\hline Unknown & $10(29 \%)$ \\
\hline \multicolumn{2}{|l|}{ Vaccine Type } \\
\hline Biken & $1(3 \%)$ \\
\hline GSK VZV Vaccines & $2(6 \%)$ \\
\hline Merck VZV Vaccines & $20(59 \%)$ \\
\hline Oka, unspecified & $3(9 \%)$ \\
\hline Okavax & $1(3 \%)$ \\
\hline Multiple VZV Vaccine Types & $7(21 \%)$ \\
\hline
\end{tabular}

${ }^{a}$ Two were published prior to vaccine licensure, one from Japan and other from the United States.

Expert Rev Vaccines. Author manuscript; available in PMC 2017 August 04. 
${ }^{b}$ Data are from Merck's Varicella Zoster Virus Identification post-marketing safety surveillance program in European countries; report by country not available.

${ }^{c}$ These 2 articles described reports of hospitalized breakthrough varicella in persons who had received one or two doses of varicella vaccine. 
Table 2

Breakthrough Varicella with Disseminated Varicella-Zoster Virus (VZV) Infection with Organ Involvement in Addition to Skin

\begin{tabular}{|c|c|c|c|}
\hline Complication Category & \# Articles & $\begin{array}{l}\text { \# Breakthrough } \\
\text { Varicella Cases }\end{array}$ & Diagnoses \\
\hline Pneumonia & $5^{15,23-26}$ & $8-9^{b, c}$ & Pneumonia \\
\hline Neurologic & $11^{10,15,18,23,24,27-32}$ & $18-24^{b, f}$ & $\begin{array}{c}\text { Meningitis, Encephalitis, Acute cerebellar ataxia, } \\
\text { Transverse myelitis, Cerebellitis }\end{array}$ \\
\hline Hematologic & $9^{15,16,18,23,26,28,34-36}$ & $10-11^{b, c, d, e}$ & $\begin{array}{c}\text { Neutropenia, Thrombocytopenia, Pancytopenia, } \\
\text { possible Macrophage activation syndrome, } \\
\text { Hemorrhagia, Disseminated intravascular } \\
\text { coagulation }\end{array}$ \\
\hline Ocular & $5^{23,24,34,37,38}$ & $5^{d}$ & $\begin{array}{l}\text { Keratitis, Conjunctivitis, Subconjunctival } \\
\text { hemorrhage, Retinitis, Blurred vision, Hemorrhagic } \\
\text { retinitis }\end{array}$ \\
\hline Renal & $2^{11,39}$ & 2 & Gross hematuria, Nephrotic syndrome \\
\hline Hepatic & $4^{18,36,40,41}$ & $3^{e}$ & Hepatomegaly, Hepatitis, Hepatic necrosis \\
\hline Secondary infections and Sepsis & $7^{16,18,23,24,35,36,42}$ & $8^{e, f}$ & $\begin{array}{l}\text { Specific organism that caused sepsis was reported } \\
\text { in } 3 \text { cases (Staph aureus in } 1 \text { case and Group A } \\
\text { streptococcus in } 2 \text { cases) }\end{array}$ \\
\hline Other & $4^{24,27,35,43}$ & $4^{f}$ & $\begin{array}{c}\text { Hypotension, Transient hearing loss, Myocarditis, } \\
\text { Multi-organ failure }\end{array}$ \\
\hline Deaths & $8^{16,18,23,24,30,36,41,43}$ & $6^{e, g}$ & \\
\hline $\begin{array}{l}\text { Total unique case-patients with any } \\
\text { of the complications above }\end{array}$ & 25 & $52-60$ & \\
\hline
\end{tabular}

${ }^{a}$ A breakthrough case may have had multiple clinical features and is included in more than one organ system category.

$b_{\text {It }}$ is cases in each of the respective complication categories, which is based on our determination of the information presented in the articles.

$c_{\text {The case described by Pillegi et al }}{ }^{26}$ developed pneumonia and hematologic complications and was included in both complication categories.

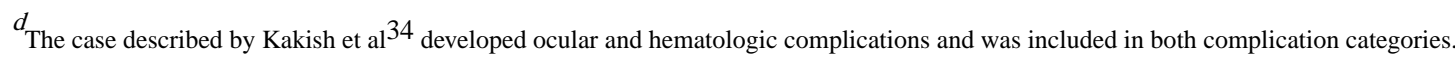

$e_{\text {The case described by Sharrar et al }}{ }^{18}$ and Wise et al ${ }^{36}$ developed hepatic complications and sepsis, and ultimately died; this case was included under all 3 complication categories. The case described by Galea et al ${ }^{24}$ and Leung et al ${ }^{16}$ developed hematologic complications and sepsis, and ultimately died; she was included in all 3 complication categories.

$f_{\text {The case described by Kwak et al }}{ }^{35}$ who developed sepsis also developed hypotension. The case described by Schwab et al 27 with neurologic complications also developed transient hearing loss.

${ }^{g}$ All deaths were previously included in at least one of the other complication categories. 